\title{
Aerodynamic and Stability Analysis of Blended Wing Body Aircraft
}

\author{
Sanjiv Paudel, Shailendra Rana, Saugat Ghimire, Kshitiz Kumar Subedi, Sudip Bhattrai* \\ Department of Mechanical Engineering, Institute of Engineering-Central Campus, Tribhuvan University, Lalitpur, Nepal
}

Email address:

mechesanjiv@gmail.com (S. Paudel), ranashailendra74@gmail.com (S. Rana), saugatghimire014@hotmail.com (S. Ghimire), kshitiz.subedi@hotmail.com (K. K. Subedi),sudip@ioe.edu.np (S. Bhattrai)

${ }^{*}$ Corresponding author

\section{To cite this article:}

Sanjiv Paudel, Shailendra Rana, Saugat Ghimire, Kshitiz Kumar Subedi, Sudip Bhattrai. Aerodynamic and Stability Analysis of Blended Wing Body Aircraft. International Journal of Mechanical Engineering and Applications. Vol. 4, No. 4, 2016, pp. $143-151$. doi: 10.11648/j.ijmea.20160404.12

Received: May 17, 2016; Accepted: June 12, 2016; Published: June 23, 2016

\begin{abstract}
The main purpose of the paper is to study the aerodynamic and stability characteristics of a blended-wing-body (BWB) aircraft. This paper presents the estimation and selection of aircraft design parameters, planform design, reflex airfoils, and conduct thorough stability investigation of the aircraft. A conceptual design of BWB aircraft has been done and the design was analyzed and refined to achieve static stability. The CFD analysis of the BWB aircraft was done at three different values of angle-of-attack (AOA) and thus the stall AOA was determined from the computational results. The dynamic stability of the aircraft has been studied under five modes namely- short period, phugoid, Dutch-roll, roll and spiral. The static stability has been achieved with a wide positive value of static margin. Results also show that the aircraft is dynamically stable for longitudinal and lateral modes when subjected to disturbances in respective conditions. The BWB aircraft fulfils the criteria of Class I Category B aircraft and shows flight level 1 characteristics in all stability modes.
\end{abstract}

Keywords: Blended-Wing-Body Aircraft, Reflex Airfoils, Static Stability, Dynamic Stability, Stability Modes

\section{Introduction}

The need and requirements of aircrafts for transporting passengers and cargo from one destination to another has took-off to greater heights since few decades. The conventional aircraft with the external wings as the major contributor of the lift are the available medium for fulfilling such purpose. Various technological developments have took off in various sectors of aircraft design such as propulsion, aerodynamics, avionics, etc. BWB aircraft being one of the promising aircraft shows a greater efficiency in its aerodynamic parameters. Though the name BWB actually famed in the aviation industry only a decade or two before, but the context has been flying in the sky since 1933, when Horten brothers conceived their first glider [1]. Recently, NASA and Boeing are continuously producing prototypes of BWB, upgrading one after the other with X48C being the recent advancement in the design [2].

The BWB aircraft is able to catch many eyes of aviation industry due to various advantages with which it overpowers over the conventional aircraft. With no clear distinction between wing-fuselage, it shows an advantage with the interference drag. Having the center body as one of the section of the airfoil, it acts as a lift generating surface, giving a higher lift-to-drag ratio. The rear end positioning of the engine gives significant reduction in noise [2]. Various design challenges have been seen in the design of BWB aircraft like being less favorable for internal cabin pressurization as a tubular body is best suited for such pressurization purpose. Having the position of control surface at the rear end adds an extra demerit making the aircraft stability design more complex. The design procedures for the design of an aircraft are similar in many cases. However, it varies on the parameters estimation like wing loading, aspect ratio and few others which ought to change upon the stability and CFD analysis. The works mainly include two sections: one section covers the basic design of the aircraft from parameter estimation to CFD analysis and section two includes the design of control 
surfaces along-with the static and dynamic stability of the aircraft.

This paper aims to give the detail procedure of an aircraft design, from initial parameter estimation to computational analysis and its stability analysis. Many researches have been done in blended-wing-body as stated, especially in planform shape optimization. This paper intends to provide the new researchers an entire basic procedure of an aircraft design from parameter selection to computational analysis through stability analysis. ANSYS ICEM CFD 14.5 is used for mesh generation and ANSYS Fluent 14.5 is used for the simulation during the entire phase of research.

\section{Literature Review}

There have been significant developments in the field of BWB research over time and it's still ongoing. Significant researches have already been put by Aerospace researchers and scientists. R. H Liebeck introduced the concept of blending the wing, fuselage, and the engines into a single lifting surface. Liebeck's design concept offered biggest improvement in aerodynamic efficiency as it reduced surface area and thereby reducing skin friction drag. His design shows that $33 \%$ reduction in surface is achievable, representing a potential revolution in subsonic transport efficiency. In his paper, BWB is considered to have an 800 passenger capacity, cruising at Mach 0.85 and a $7000 \mathrm{~nm}$ range [3]. In 2002, Qin presented the main aerodynamic advantages of the new BWB design are its lower wetted area to volume ratio and lower interference drag as compared to the conventional aircraft. Indeed, an increase in lift to drag maximum of about $20 \%$ over the conventional design has been estimated for the BWB [4]. In 2003, Roman studied aerodynamics of high subsonic BWB configurations, concluded that Mach number 0.93 has penalty performance relative to Mach number 0.85 [5]. Later in 2004, Qin again calculated the aerodynamic performance of BWB aircraft; they carried out 3-D aerodynamic surface optimization of different BWB configurations and improved aerodynamic performance at cruise condition [6]. Toshihiro Ikeda designed a conceptual model of BWB, wingspan of $79.8 \mathrm{~m}$, wetted aspect ratio 181 and wetted surface area $3524.85 \mathrm{~m}^{2}$, capable of carrying 555 passengers [7]. HAW students conducted research study of AC 20.30 BWB aircraft whose geometry is based on VELA 2 [8]. In their research, MH45 airfoil was employed in wing profile whereas the body profile was equipped with MH91 airfoil [9]. DLR-LY-BWB configuration, whose mission requirements were a range of $7560 \mathrm{~nm}$ and 500 seat capacities, was designed by DLR that resulted from the studies presented in [10] and [11]. NASA successfully completed the flight of X-48C aircraft, a modified version of $\mathrm{X}-48 \mathrm{~B}$, in 2013 to investigate and validate the aerodynamic characteristics of the Blended Wing Body Aircraft design concept [12].

\subsection{Initial Aircraft Sizing}

The primary design parameters for the BWB aircraft studied in this paper taken into consideration are wing loading, Take-off-weight and engine thrust (or engine power). Wing loading affects stall speed, climb rate, take-off and landing distances, and turn performance [13]. The wing loading determines the design lift coefficient, and impacts drag through its effect upon wetted area and wing span. The aircraft weight and wing planform area is initially taken intuitively, which is supposed to be changed to the actual required weight and size upon stability analysis further in this paper.

The weight of the aircraft is initially chosen to be $2.51 \mathrm{~kg}$ considering the weight of a model aircraft. The values from 4.83 $\mathrm{kg} / \mathrm{m}^{2}$ to $8.78 \mathrm{~kg} / \mathrm{m}^{2}$ is an acceptable range for the wing loading [14]. Using the wing loading of $5.4 \mathrm{~kg} / \mathrm{m}^{2}$, the planform area of the aircraft is calculated to be $0.4648 \mathrm{~m}^{2}$ using:

Planform area $=$ Weight of the aircraft / Wing Loading

The necessary deflection of flaps upon stability analysis slightly and changes the planform area of the aircraft, which does not change the overall aircraft characteristics like lift and drag significantly.

\subsection{Operational and Geometrical Parameters}

Out of the large number of reflex airfoils to compensate for the lack of horizontal stabilizers, four airfoils namely MH45, MH62, SB97 and HS522 $(\bmod 14 \%)$ were analyzed in XFLR5 at Reynolds number 400000 and their polar characteristics were compared with each other. 400,000 Reynolds number was randomly selected to study and compare the aerodynamic characteristics of airfoils under consideration.

Table 1. Characteristics of different airfoils.

\begin{tabular}{llllll}
\hline Airfoil & $\mathbf{C}_{\mathbf{I m a x}}$ & $\boldsymbol{\alpha}_{\max }$ & $\mathbf{C l} / \mathbf{C d})_{\boldsymbol{a m a x}}$ & $\mathbf{C d}_{\text {amax }}$ & $\mathbf{C m}_{\text {amax }}$ \\
\hline HS522 14\% & 1.35 & 140 & 42 & 0.032 & 0.019 \\
MH45 & 1.23 & 11.50 & 46.6 & 0.026 & 0.006 \\
MH62 & 1.15 & 10.50 & 42.5 & 0.027 & 0.001 \\
SB97 & 1.27 & 120 & 36.2 & 0.035 & -0.005 \\
\hline
\end{tabular}

From the results that follow, MH62 was rejected early because of its low maximum lift coefficient and small maximum angle of attack as compared to HS522-14\% and MH45, which can be seen from table 1. SB97 was then rejected due to the low value of lift to drag ratio and high drag coefficient near stall angle. Also, it has negative moment coefficient which is unfavorable considering the design of our aircraft. Due to fairly high lift to drag ratio near stall angle, high stall angle and fairly constant moment coefficient curve throughout the range of angle of attack, the other two airfoils were selected. Out of these two airfoils, the HS522 $(\bmod 14 \%)$ was chosen to be used at the root section because of its thickness and MH45 towards the tip providing the aerodynamic twist. The airfoil coordinates are taken from the Hartmut Siegmann airfoil database [15].

\subsection{Aircraft Planform Design}

The initial planform is assumed to be trapezoidal in shape. 
Table 2 lists the conceptual design parameters as below:

Table 2. Major parameters of Aircraft Planform.

\begin{tabular}{lll}
\hline S.N. & Parameters & Value \\
\hline 1. & Wing loading & $5.40 \mathrm{~kg} / \mathrm{m}^{2}$ \\
2. & Maximum take-off weight & $2.51 \mathrm{~kg}$ \\
3. & Aspect ratio (AR) & 5.78 \\
4. & Reference area (S $\left.\mathrm{S}_{\text {ref }}\right)$ & $0.4648 \mathrm{~m}^{2}$ \\
5. & Wing span (b) & $1.8 \mathrm{~m}$ \\
6. & Leading edge sweep angle & $33^{0}$ \\
7. & Main (root) chord & $760 \mathrm{~mm}$ \\
8. & Tip chord & $100 \mathrm{~mm}$ \\
\hline
\end{tabular}

\subsection{Calculation of Level Flight Condition}

Table 3 lists the major characteristics of the BWB aircraft as below:

Table 3. Characteristics of BWB aircraft.

\begin{tabular}{lll}
\hline S.N. & Characteristics & Value \\
\hline 1. & Default AOA & $3^{0}$ \\
2. & Lift coefficient(CL) & 0.5 \\
3. & Flight velocity & $12.8 \mathrm{~m} / \mathrm{s}$ \\
4. & Reynolds number(tip) & 86,667 \\
5. & Reynolds number(root) & $6,58,667$ \\
\hline
\end{tabular}

\subsection{Construction of Model Geometry}

The wing planform of the whole aircraft is drawn with the root chord and tip chord equal to $760 \mathrm{~mm} \mathrm{HS}-522(\bmod 14 \%)$ and $100 \mathrm{~mm} \mathrm{MH}-45$ respectively in XFLR5. $450 \mathrm{~mm}$ HS$522(\bmod 14 \%)$ airfoil at $200 \mathrm{~mm}$ from root chord and 280 $\mathrm{mm}$ MH-45 airfoil at $350 \mathrm{~mm}$ from root chord are placed in the geometry. Dihedral angle and twist are chosen based on historical research and there is a $2^{0}$ dihedral and $-3^{0}$ twist from root chord to chord at $200 \mathrm{~mm}$, and a $2^{0}$ dihedral and $3^{0}$ twist from the chord at $200 \mathrm{~mm}$ to chord at $350 \mathrm{~mm}$. The model of the aircraft designed using the calculations above is shown in Figure 1.

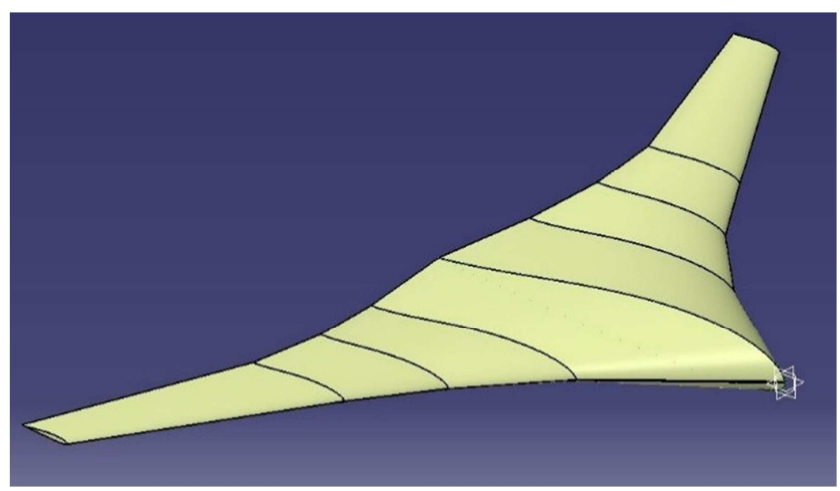

Figure 1. BWB aircraft model geometry.

\subsection{Numerical Methods}

For the modeling of the 3-D incompressible flow, the solvers resolved the mass and momentum conservation equations. The mass, or continuity equation can be written as:

$$
\frac{\partial \rho}{\partial t}+\nabla \cdot(\rho \vec{v})=0
$$

The above equation reduces to $\nabla \cdot \vec{v}=0$ for incompressible fluid.

The transport of momentum in an inertial reference frame is given by:

$$
\frac{\partial}{\partial t}(\rho \vec{v})+\nabla \cdot(\rho \vec{v} \vec{v})=-\nabla p+\nabla \cdot(\overline{\bar{T}})+\rho \vec{g}+\vec{F}
$$

Where, $p$ is the static pressure, $\overline{\bar{T}}$ is the stress tensor, and $\rho \vec{g}$ and $\vec{F}$ are the gravitational body force and external body forces respectively.

Pressure-velocity coupled solution method was used for both the 2-D and 3-D analyses. A least square cell based method was used for gradient calculation with second order upwind extrapolation for primitive variables in the momentum solution. The courant number used for the coupled solution is unity. K- $\omega$ SST model is used to model the turbulence, based on the type of flow and flow separation near the boundary wall, with SST k- $\omega$ being more accurate and reliable for flow with adverse pressure gradients.

The transport equations for $S S T k-\omega$ model are:

$$
\frac{\partial}{\partial t}(\rho k)+\frac{\partial}{\partial x_{i}}\left(\rho k u_{j}\right)=\frac{\partial}{\partial x_{j}}\left(\Gamma_{k} \frac{\partial k}{\partial x_{j}}\right)+G_{k}-Y_{k}+S_{k}
$$

And,

$$
\frac{\partial}{\partial x}(\rho \omega)+\frac{\partial}{\partial x_{j}}\left(\rho \omega u_{j}\right)=\frac{\partial}{\partial x_{j}}\left(\Gamma_{\omega} \frac{\partial \omega}{\partial x_{j}}\right)+G_{\omega}-Y_{\omega}+D_{\omega}+S_{\omega}
$$

In these equations, $G_{k}$ represents the generation of turbulence kinetic energy due to mean velocity gradients. $G \omega$ represents the generation of $\omega . \Gamma k$ and $\Gamma \omega$ represent the effective diffusivity of $k$ and $\varepsilon$ respectively. $Y k$ and $Y \omega$ represent the dissipation of $k$ and $\omega$ due to turbulence. $D \omega$ represent the cross diffusion-term. $S k$ and $S \omega$ are user defined source terms. Both turbulent kinetic energy and specific dissipation rate used first order upwind for the solution.

\subsection{Full Body CFD Analysis}

The two reflex airfoils MH45 and HS522 -14\% are used in the full body BWB. The analysis of the airfoils are done at two AOA, $3^{0}$ and $7^{0} .3^{0}$ being the default AOA of the aircraft and $7^{\circ}$ AOA, which is the angle at which the aircraft attains its maximum lift to drag ratio. At $3^{\circ}$ and $7^{0}$, the 2D airfoil analysis is carried out to visualize airfoil characteristics like pressure distribution, velocity distribution, lift force, and drag force. The computation domain consists of a $10 \mathrm{~m}$ by $10 \mathrm{~m}$ control volume, with an airfoil with $760 \mathrm{~mm}$ chord. The pressure contours for both the airfoils at default AOA $3^{0}$ are shown in Figure 2 and 3 respectively and the corresponding values of lift and drag at $3^{0}$ and $7^{0}$ are listed in Table 4. 


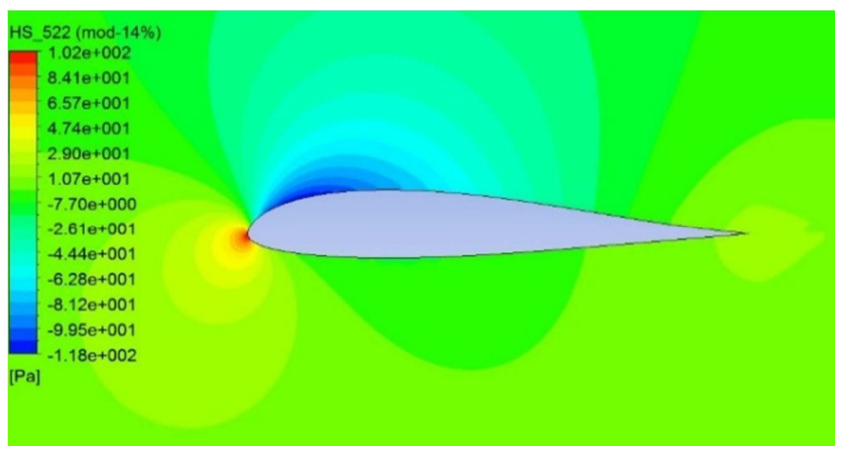

Figure 2. Pressure contour of HS-522 mod-14.

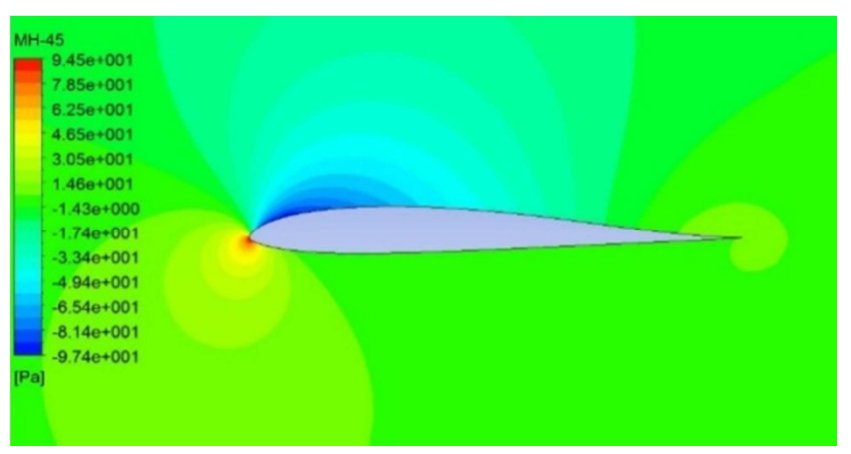

Figure 3. Pressure contour of $M H-45$.

The pressure contour plots of HS522 and MH45 airfoils are shown in above figures, which shows the stagnation point of airfoil just below the leading edge at an angle of $3^{0}$. The aircraft generates take off lift at an angle of $3^{0}$, which is thus the default angle of attack of the aircraft. Fig. 2 shows a lower pressure region of $-118 \mathrm{~Pa}$ at the upper surface and $\mathrm{a}$ higher pressure region of $102 \mathrm{~Pa}$ at the lower surface of HS522 airfoil, thus generating a lift to drag ratio of 42 . Fig. 3 shows a lower pressure region of-97.4 $\mathrm{Pa}$ at the upper surface and a higher pressure region of $94.5 \mathrm{~Pa}$ at the lower surface of MH45, thus generating a lift to drag ratio of 46.6.

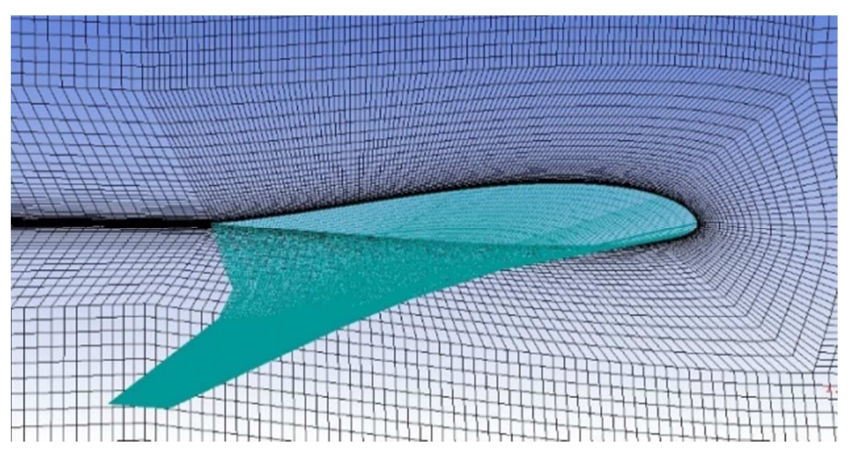

Figure 4. Mesh across the blended wing body aircraft.

Table 4. Lift and drag values at $3^{\circ}$ and $7^{\circ} \mathrm{AOA}$.

\begin{tabular}{lllll}
\hline Airfoil & AOA & $\mathbf{C}_{\mathbf{l}}$ & $\mathbf{C}_{\mathbf{d}}$ & $\mathbf{C}_{\mathbf{l}} / \mathbf{C}_{\mathbf{d}}$ \\
\hline \multirow{2}{*}{ HS 522 14\%- modified } & $3^{0}$ & 0.414 & 0.012 & 34.50 \\
& $7^{0}$ & 0.812 & 0.018 & 45.11 \\
\multirow{2}{*}{ MH 45 } & $3^{0}$ & 0.483 & 0.014 & 34.50 \\
& $7^{0}$ & 0.863 & 0.018 & 47.94 \\
\hline
\end{tabular}

The unstructured mesh of the half-body BWB aircraft shown in Figure 4, has a density of $1,35,472$ cells. The dimensionless $\mathrm{y}+$ value of $1 \mathrm{~mm}$ is used to find the corresponding minimum wall distance at Reynolds number 658,667 , which is $0.024 \mathrm{~mm}$.

The computation domain for the half body simulation extends to $16 \mathrm{~m}$ upstream and $16 \mathrm{~m}$ downstream from leading edge and trailing edge of the root chord respectively. The CFD analysis of the BWB aircraft is done for two angle of attack, one at its default angle of attack at

3 degrees, and 7 degrees, where the aircraft has maximum lift to drag ratio $\left(\mathrm{C}_{\mathrm{L}} / \mathrm{C}_{\mathrm{D}}\right)$. Results for the full body simulation are shown in the Figure 5 and 6 and the obtained aerodynamic parameters are listed in table 5 .

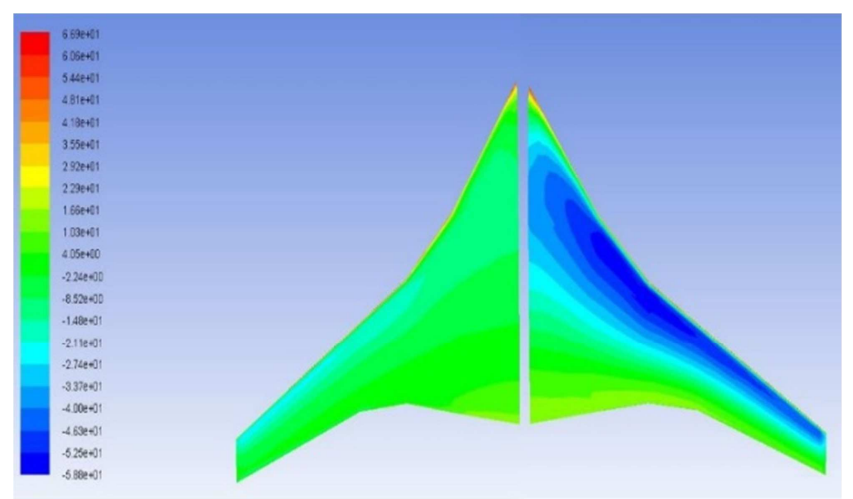

Figure 5. Pressure contour of BWB in lower (left) and upper (right) region at $3^{0}$.

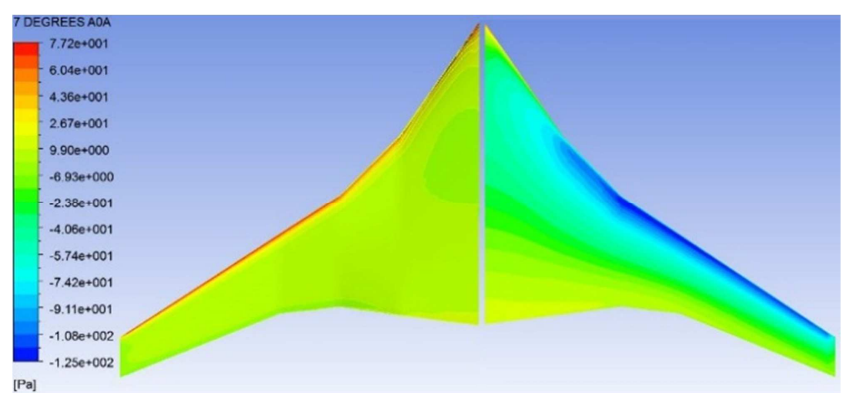

Figure 6. Pressure contour of BWB in lower (left) and upper (right) region at $7^{\circ} A O A$.

The pressure contours of BWB aircraft at 3 degrees AOA shows a lower pressure region of $-58.6 \mathrm{~Pa}$ at upper body and $46 \mathrm{~Pa}$ at its lower body, which gives a $\mathrm{C}_{\mathrm{L}} / \mathrm{C}_{\mathrm{D}}$ ratio of $\mathrm{BWB}$ aircraft of 8.87 . Further, the ratio increases linearly up to 7 degrees AOA which shows a higher pressure region of $26 \mathrm{~Pa}$ at its lower body and $-125 \mathrm{~Pa}$ at its upper body, which gives a $\mathrm{C}_{\mathrm{L}} / \mathrm{C}_{\mathrm{D}}$ ratio of $\mathrm{BWB}$ aircraft of 16.45 . Beyond this angle of attack, the body shows a tendency of shock wave generation spoiling the lift. Hence, 7 degrees AOA is considered to be the AOA of maximum lift to drag ratio of the BWB aircraft.

Table 5. Showing lift and drag values of $B W B$ at $3^{\circ}$ and $7^{\circ} A O A$.

\begin{tabular}{lllll}
\hline S.N. & Angle of attack & $\mathbf{C}_{\mathbf{L}}$ & $\mathbf{C}_{\mathbf{D}}$ & $\mathbf{C}_{\mathbf{L}} / \mathbf{C}_{\mathbf{D}}$ \\
\hline 1. & $3^{0}$ & 0.1751 & 0.01975 & 8.87 \\
2. & $7^{0}$ & 0.51 & 0.031 & 16.45 \\
\hline
\end{tabular}




\section{Stability Analysis of the BWB Aircraft}

\subsection{Sizing of Control Surfaces}

The three primary control surfaces in the aircraft namelyflaps, rudders and elevons as shown in Figure 7, are chosen based on the required mitigation of disturbances. The sizing of the control surfaces are obtained iteratively to balance the input disturbance parameters within acceptable damping time. Table 6 lists the sizing and characteristics of the control surfaces.

Table 6. Size of different control surfaces and their respective locations.

\begin{tabular}{llllll}
\hline Control Surfaces & C1 (m) & C2 (m) & Span $(\mathbf{m})$ & Surface area $\left(\mathbf{m}^{2}\right)$ & Airfoil \\
\hline Flaps & 0.107 & 0.07 & 0.131 & 0.02318 & MH45 \\
Elevons & 0.065 & 0.029 & 0.444 & 0.02086 & MH45 \\
Rudders & 0.2 & 0.08 & 0.18 & 0.0504 & NACA 0009 \\
\hline
\end{tabular}

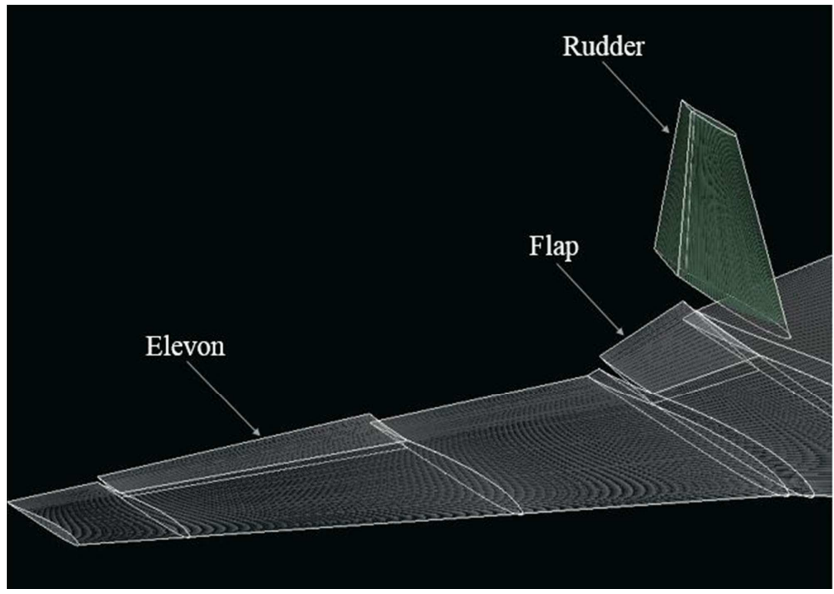

Figure 7. 3-d view of control surfaces.

\subsection{Static Stability of the Aircraft}

After the mass components like ducted fan motor, electronic speed controller (ESC), battery, etc. were provided at their estimated positions, the computed position of the $\mathrm{C}$. G. was found to lie at $421.225 \mathrm{~mm}$ and the neutral point of the aircraft was found to be at $495.96 \mathrm{~mm}$.

With mean aerodynamic chord (MAC) of $415.671 \mathrm{~mm}$, the static margin is obtained as below:

$$
\mathrm{S} . \mathrm{M}=\frac{\mathrm{X}_{\mathrm{np}}-\mathrm{X}_{\mathrm{cg}}}{\mathrm{MAC}}=\frac{495.96-421.225}{415.671}=18 \%
$$

Such positive static margin shows that the aircraft is statically stable, as shown in the results for static longitudinal stability of the aircraft. Figure 8 shows the plot of $\mathrm{Cm}$ against angle of attack, denoted by alpha, with the pitching moment coefficient obtaining negative value near AOA of 80. This implies the aircraft has a stick-free nose down tendency at high angle of attack.

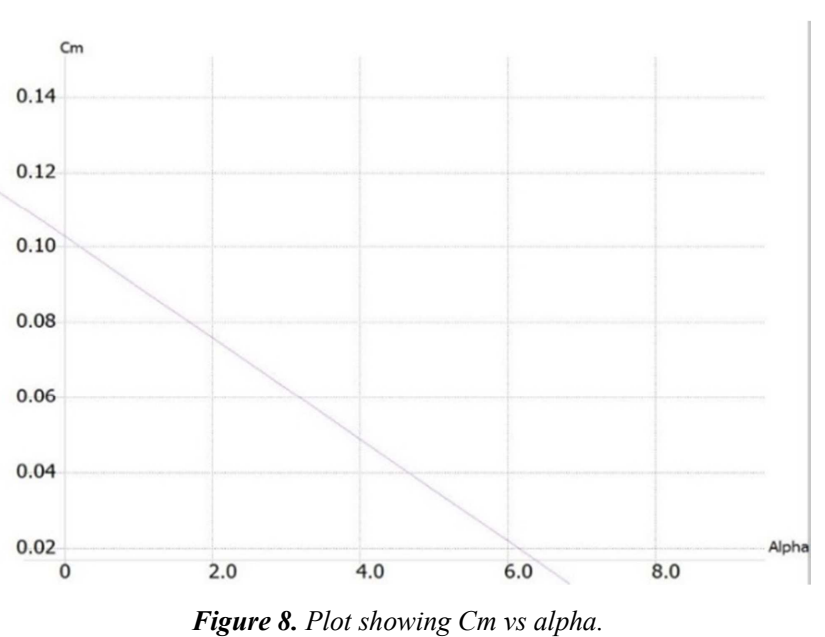

\subsection{Dynamic Stability}

Longitudinal and lateral stability of the aircraft have been studied. For longitudinal motion, dynamic stability has been studied under two modes namely: short period and Phugoid mode whereas, for lateral motion, dynamic stability has been studied under three modes namely: Dutch-roll, roll and spiral.

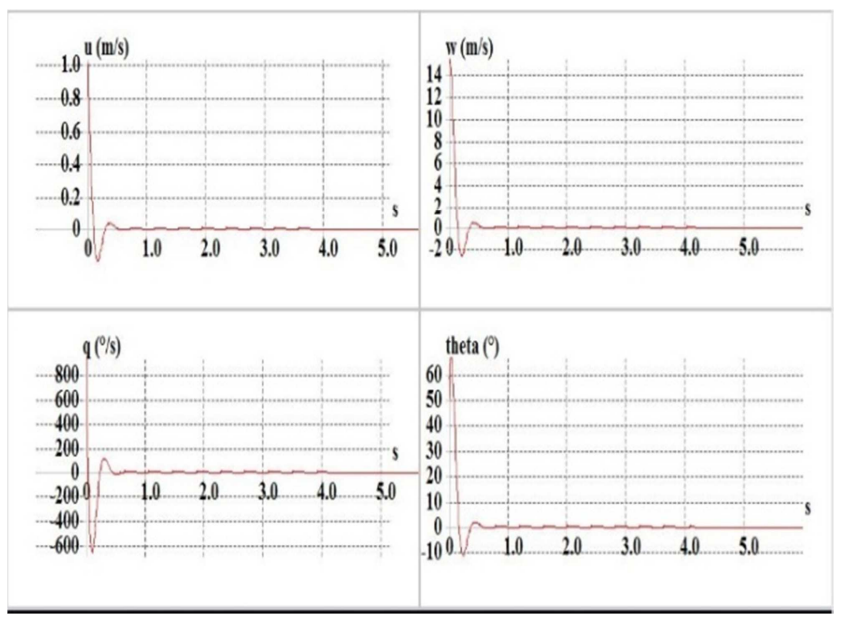

Figure 9. Time response for short period mode. 


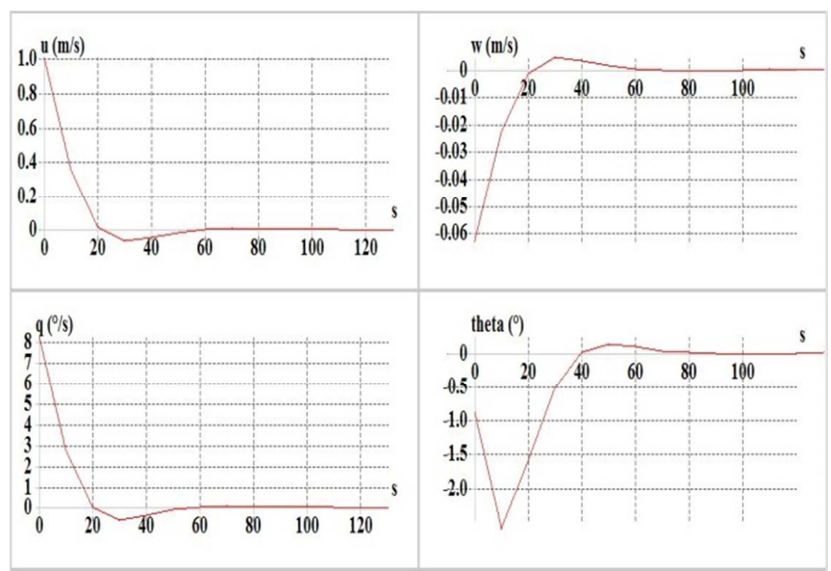

Figure 10. Time response for phugoid mode.

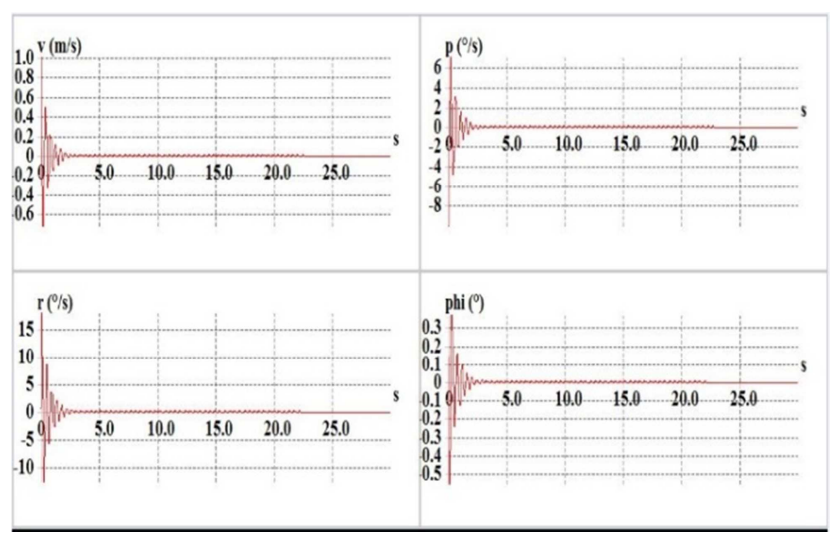

Figure 11. Time response for Dutch-roll mode.

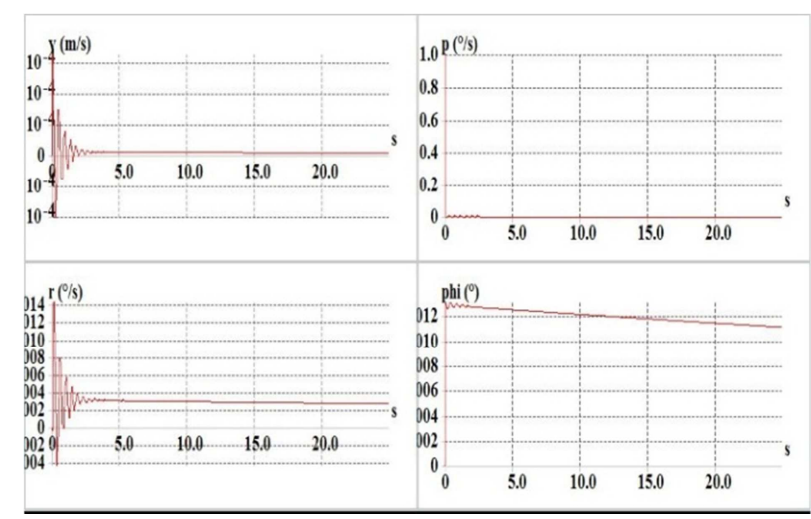

Figure 12. Lateral time response for initial disturbance of $1^{\circ} \mathrm{s}^{-1}$ in roll rate.

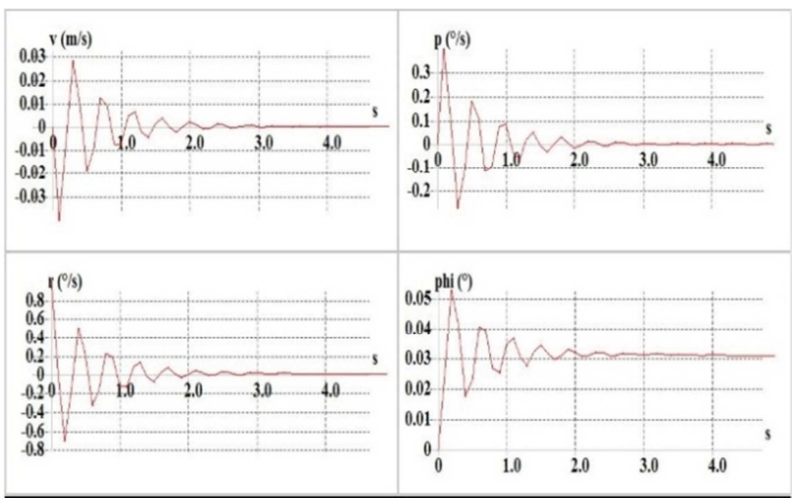

Figure 13. Lateral time response for initial disturbance of $1{ }^{\circ} \mathrm{s}^{-1}$ in yaw rate.

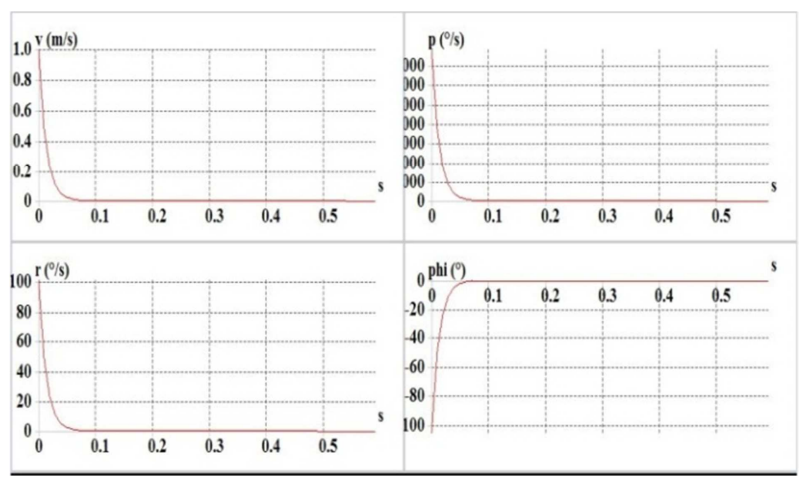

Figure 14. Time response for roll mode.

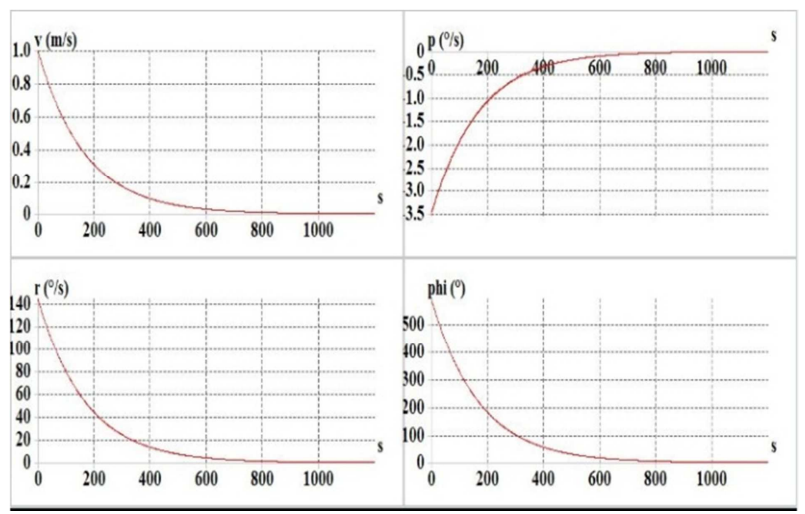

Figure 15. Time response for spiral mode.

\subsection{Dynamic Stability of the BWB for Longitudinal Motion}

For longitudinal motion, the investigation of the dynamic stability of the BWB requires longitudinal state matrix. The two main stability modes for the longitudinal motion are the Short Period and the Phugoid mode. Two different sets of conjugate eigenvalues are obtained from the longitudinal state matrix which represent the two longitudinal stability modes.

From XFLR computation, the eigenvalues obtained from the longitudinal state matrix are as follows:

$$
\begin{aligned}
& \lambda_{1}=-8.7623+15.3862 \mathrm{i} \\
& \lambda_{2}=-8.7623-15.3862 \mathrm{i} \\
& \lambda_{3}=-0.0733+1.1807 \mathrm{i} \\
& \lambda_{4}=-0.0733-1.1807 \mathrm{i}
\end{aligned}
$$

The longitudinal state matrix consists of longitudinal stability derivatives. After the computation of longitudinal stability derivatives, the longitudinal state matrix $\mathrm{A}$ is obtained as below:

$$
\mathrm{A}=\left[\begin{array}{cccc}
-1.14781 & 0.108506 & -40.0081 & -9.81 \\
-13.062 & -74.7506 & 3.37807 & 0 \\
5.27001 & -0.980376 & -2.13086 & 0 \\
0 & 1 & 0 & 0
\end{array}\right]
$$

The characteristic equation is obtained from the expansion of the following determinant: 


$$
|\lambda I-\mathrm{A}|
$$

Where, I is the identity matrix.

\subsubsection{Short Period Mode}

This mode consists of a symmetric disturbance caused due to sudden up-gust or step elevator which changes the angle of incidence and results in rapid pitching of the aircraft about the center of gravity. Conjugate eigenvalues $\lambda_{1}$ and $\lambda_{2}$ belong to the Short Period mode. Since the real parts of the Short Period mode eigenvalues $\lambda_{1}$ and $\lambda_{2}$ are negative, it can be predicted that the plane is inherently stable in Short Period mode. The Short Period poles are underdamped with a natural frequency $\omega_{n_{S}}$ of $17.7016 \mathrm{rads}^{-1}$ and a damping ratio $\zeta_{s}$ of 0.495 . The positive damping ratio indicates that the aircraft is able to damp out the short period mode by itself, bringing it back to a stable longitudinal position. Figure 9 represents the time response for the short period mode. It can be observed that the short period mode shows a rapidly under-damped response as evidenced by a small time period of 0.4084 secs. The time taken to halve amplitude of the disturbances is 0.08 secs. The forward speed $u$, downward velocity $w$, pitch rate $q$ and pitch angle $\theta$ are analyzed to observe their time response in this mode. It is seen that all the four parameters i.e. forward speed, downward velocity, pitch rate and pitch angle rapidly recover to equilibrium.

\subsubsection{Phugoid Mode}

This mode is also excited by the same symmetric disturbance and consists of slow sinusoidal motion where there is large amplitude variation of air-speed, pitch angle and altitude, with no variation in angle of attack. The next conjugate eigenvalues $\lambda_{3}$ and $\lambda_{4}$ belong to the Phugoid mode. The real parts of the Phugoid mode eigenvalues $\lambda_{3}$ and $\lambda_{4}$ are negative, implying that the plane is inherently stable in Phugoid mode. The Phugoid mode poles are also under-damped with a natural frequency $\omega_{n_{p}}$ of 1.1823 rads $^{-}$ ${ }^{1}$ and a damping ratio $\zeta_{p}$ of 0.062 . The positive damping ratio indicates that the aircraft is able to damp out the Phugoid mode by itself, bringing it back to a stable longitudinal position. Figure 10 represents the time response for the Phugoid mode. It is seen that the Phugoid mode exhibits a slow under-damped response as evidenced by a large time period of 5.3216 secs. The time taken to halve the amplitude of the disturbances in this mode is 9.4543 secs. Similar to the Short Period mode, the forward speed $u$, downward velocity $w$, pitch rate $q$ and pitch angle $\theta$ are analyzed to observe their time response in this mode. It can be observed that all the four parameters i.e. forward speed, downward velocity, pitch rate and pitch angle slowly attain equilibrium.

\subsection{Dynamic Stability of the BWB for Lateral Motion}

The investigation of the dynamic stability of the BWB for lateral motion requires lateral state matrix. For lateral motion of the aircraft, there are three main stability modes. They are roll mode, Dutch-roll mode and spiral mode. Those eigenvalues correspond to respective lateral modes. The eigenvalues obtained from the lateral state matrix from calculation are as follows:

$$
\begin{aligned}
& \lambda_{1}=-1.6103+14.8033 \mathrm{i} \\
& \lambda_{2}=-1.6103-14.8033 \mathrm{i} \\
& \lambda_{3}=-74.8026 \\
& \lambda_{4}=-0.0059
\end{aligned}
$$

The lateral state matrix consists of lateral stability derivatives. After the computation of lateral stability derivatives, the lateral state matrix $\mathrm{A}$ is obtained as below:

$$
\mathrm{A}=\left[\begin{array}{cccc}
-1.14781 & 0.108506 & -40.0081 & -9.81 \\
-13.062 & -74.7506 & 3.37807 & 0 \\
5.27001 & -0.980376 & -2.13086 & 0 \\
0 & 1 & 0 & 0
\end{array}\right]
$$

Similar to that of longitudinal motion, a characteristic equation is obtained, from which the eigenvalues for lateral motion are determined as below:

$$
|\lambda I-\mathrm{A}|
$$

Where, I is the identity matrix.

\subsubsection{Dutch Roll Mode}

The Dutch-roll mode consists of an oscillatory combined rolling and yawing motions of the aircraft. The eigenvalues $\lambda_{1}$ and $\lambda_{2}$ correspond to the Dutch-roll mode of the BWB aircraft. The real negative parts of the Dutch-roll mode eigenvalues signifies that the aircraft is inherently stable in Dutch-roll mode. The Dutch-roll poles are under-damped with a natural frequency $\omega_{n_{D}}$ of $10.7435 \mathrm{rads}^{-1}$ and a damping ratio $\zeta_{D}$ of 0.108 . The positive damping ratio indicates that the aircraft is able to damp out the Dutch-roll mode by itself without the help of external control inputs and brings it back to a stable lateral position. However, due to small damping ratio, the disturbance is not sufficiently damped. Figure 11 represents the time response for the Dutch-roll mode. The side-slip velocity $v$, roll rate $p$, yaw rate $r$ and bank angle $\phi$ are analyzed to observe their time response in this mode. It can be seen that all the four parameters i.e. side-slip velocity, roll rate, yaw rate and bank angle slowly recover to equilibrium. Initial conditions response analysis, where an initial disturbance in either roll rate, yaw rate or side-slip velocity is given as input, was also conducted to observe the lateral time response. Figure 12 shows the lateral response to an initial disturbance of $1^{\circ} \mathrm{s}^{-1}$ in roll rate. It is observed that the disturbance in roll rate quickly decayed to zero, except side-slip velocity, yaw rate and bank angle, which gradually approached zero. However, the amplitudes of disturbance in side-slip velocity, yaw rate and bank angle is so small that their effects can be neglected. Figure 13 represents the lateral 
response to an initial disturbance of $1^{\circ} \mathrm{s}^{-1}$ in yaw rate. It is seen that the disturbance in side-slip velocity, roll rate and yaw rate vanished in about 5 secs, except bank angle, which gradually decayed to zero.

\subsubsection{Roll Mode}

Roll mode consists of the rolling motions of the aircraft. The eigenvalue $\lambda_{3}$ is associated with the roll mode, which is highly convergent and damped. Since the eigenvalue is negative, it implies that the aircraft is stable for the roll mode. The roll damping time $T_{\xi_{\text {Roll }}}$, obtained from the eigenvalue is 0.0134 secs. This value indicates that the aircraft is very stable for the roll mode and is able to damp it out itself. Figure 14 shows the plots of time response for roll mode. It is observed that the side-slip velocity, roll rate, yaw rate and bank angle recover rapidly to equilibrium.

\subsubsection{Spiral Mode}

The last eigenvalue $\lambda_{4}$ represents the spiral mode, which can be either convergent or divergent. The negative eigenvalue signifies that the aircraft is stable for the spiral mode and is convergent in nature. The time to damp to half amplitude $T_{1 / 2_{\text {spiral }}}$ is $118 \mathrm{secs}$. This value indicates that the degree of stability in spiral mode is low, so it requires special attention. Figure 15 shows the time response for spiral mode. It is seen that the side-slip velocity, roll rate, yaw rate and bank angle recover very slowly to equilibrium.

\subsection{Flight Quality of the BWB Aircraft}

The flight quality represents the degree of stability and control that is required for the pilot to keep the aircraft flyable and safe. Such flight qualities are based on the pilot's opinion of the flying characteristics of the aircraft, and is represented by empirical numeric values for classification.

\subsection{Flight Quality for the Longitudinal Motion}

According to the classification of aircraft presented in [16], the BWB aircraft is a Category B Class I aircraft. For longitudinal motion, the classification is based on the characteristic values of the damping ratio of the short period mode and the Phugoid mode. Comparing the computed values of the damping ratio of both the short period mode and the Phugoid mode with that of the values given in [16], it can be confirmed that the BWB aircraft conforms to the specifications for the level 1 definition of the flying qualities for the longitudinal motion.

\subsection{Flight Quality for the Lateral Motion}

The flight qualities of the BWB aircraft is determined with the help of Dutch-roll frequency and its damping, roll time constant and the time to double amplitude for spiral mode. For Category B Class I aircraft, the roll time constant $T_{\xi_{\text {Roll }}}=0.0134 \mathrm{~s}$ is smaller than $1.4 \mathrm{~s}$, which is the required maximum value for level 1 [16], hence the aircraft shows level 1 flying qualities for the roll mode. The computed damping ratio $\zeta_{D}$ of the BWB aircraft is 0.108 , which results in level 1 value as the minimum requirement of damping ratio $\zeta_{D}$ is 0.08 for the level 1 definition of the flight qualities [16]. The product of the Dutch-roll natural frequency and damping ratio gives the value of $\omega_{n_{D}} \zeta_{D}=$ $1.6103 \mathrm{rads}^{-1}$, which also provides the level 1 value as the minimum required value is $0.08 \mathrm{rads}^{-1}$ for level 1 definition. Hence, the flight quality for the whole Dutchroll mode is level 1. For spiral mode, the time to half amplitude is approximately $120 \mathrm{~s}$. In other words, the time to double amplitude is $30 \mathrm{~s}$, which exceeds the minimum time required to double amplitude for level 1 definition [16]. So the aircraft depicts level 1 flying qualities for the spiral mode.

\section{Conclusion}

A complete CFD analysis of the BWB aircraft was done at $3^{0}$ and $7^{0}$ AOA. Results showed a greater amount of lift to drag ratio at $7^{0} \mathrm{AOA}$ and thus the stall AOA was determined. The stability investigation of the BWB shows that the aircraft is statically stable with a positive static margin of $18 \%$. For longitudinal motion of the BWB, the aircraft is dynamically stable in all two modes namely- short period and phugoid with all the eigenvalues of the coefficient matrix having negative values for real parts. The BWB belongs to Category B Class I aircraft. The flight quality of the BWB is a function of damping ratio of the aircraft. The computed values of damping ratio for short period and phugoid mode shows a flight level 1 characteristics. For lateral motion of the BWB, the aircraft is also dynamically stable in all three modes since all the eigenvalues of the coefficient matrix for lateral motion have negative values for real parts. As for the flight quality in lateral motion, the aircraft shows flight level 1 characteristics in all three modes namely-Dutch-roll, Roll and Spiral. Overall, the BWB aircraft is a Category B Class I aircraft with level 1 flight quality in all modes.

\section{References}

[1] Bullard, D. (1997-2008). Horten Biography. Retrieved October 2015, from Douglas Bullard Website: http://www.nurflugel.com/Nurflugel/Horten_Nurflugels/Horte n_Biography/horten_biography.html

[2] NASA. (2015). NASA Website. Retrieved August 2015, from http://www.nasa.gov/centers/dryden/Features/global_observer _wing_tests.html

[3] Liebeck, R. H., Page, M. A., \& Rawdon, B. K. (1998). Blended Wing Body Subsonic Commercial Transport. AIAA Paper 98-0438.

[4] Qin, N., Vavelle, A., Le Moigne, A., Hackett, K., \&Weinerfelt, P. (2002). Aerodynamic Studies for Blended Wing Body Aircraft. AIAA.

[5] Roman, D., Gilmore, R., \& Wakayama, S. (2003). Aerodynamics of High-subsonic Blended Wing Body Configuration. AIAA Paper. 
[6] Qin, N., \&Weinerfelt, P. (2004). Aerodynamic Considerations of Blended Wing Body Aircraft. Progess in Aerospace Sciences, 40, 321-343.

[7] Ikeda, T., \& Bil, C. (2006). Aerodynamic Performance of a Blended Wing-Body Configuration Aircraft. Proceedings of the ICAS (pp. 1-10). Edinburgh: ICAS.

[8] University of Greenwich. (2015). University of Greenwich. Retrieved August 2003-2015, from https://fseg.gre.ac.uk/fire/VELA.html

[9] Scholz, D. (2007). A Student Project of a Blended Wing Body Aircraft-From Conceptual Design to Flight Testing. EWADE 2007- 8th European Workshop on Aircraft Design Education. Samara: Samara State Aviation University.

[10] Ciampa, P. D., Zill, T., Pfeiffer, T. \& Nagel, B. (2011). A Functional Shape Parametrization of Approach for Preliminary Optimization of Unconventional Aircraft. CEAS.

[11] Zill, T., Ciampa, P. \& Nagel, B. (2012). Multidisciplinary Design Optimization in a Collaborative Distributed Aircraft Design System. In 50th AIAA Aerospace Sciences Meeting.
[12] The Boeing Company. (2016). Technology: The Boeing Company. [Online] Available at: http://www.boeing.com/features/2013/04/bds-x48c-04-2413.page [Accessed 8 June 2016].

[13] Raymer, D. P. (2006). Aircraft design: A Conceptual Approach (4th Edition ed.). Virginia: American Institute of Aeronautics and Astronautics Inc.

[14] Nicolai, L. M., \& Carichner, G. E. (2010). Fundamentals of Aircraft and Airship Design, Volume 1 - Aircraft Design . California: American Institute of Aeronautics and Astronautics.

[15] Siegmann, H. (1988-2015). Aerodesign. Retrieved August 2015, from Hartmut Siegmann Website: http://www.aerodesign.de/index.htm

[16] Nelson, R. C. (1998). In Flight Stability and Automatic Control. New York/San Francisco: WCB/McGraw-Hill. 